\title{
Lithium- and Valproate-Induced Alterations in Circadian Locomotor Behavior in Drosophila
}

\author{
Mehmet E Dokucu ${ }^{*, 1,2}$, Liangping Yu ${ }^{1,2}$ and Paul H Taghert ${ }^{2}$ \\ 'Department of Psychiatry, Washington University School of Medicine, St Louis, MO, USA; 'Department of Anatomy and Neurobiology, \\ Washington University School of Medicine, St Louis, MO, USA
}

\begin{abstract}
Lithium and valproate are commonly used mood stabilizers, but their action pathways are not clearly understood. They also suffer from multiple toxic effects that limit their utility. Elucidating their action mechanisms could lead to newer agents and better understanding of the etiopathogenesis of bipolar disorder. We have expanded the study of signaling mechanisms of lithium and valproate by using Drosophila circadian locomotor activity as a robust behavioral assay that is amenable to genetic manipulations. We demonstrate that lithium affects the circadian system of Drosophila similarly to what has been reported in the mammalian studies. We show that lithium and valproate share effects on the circadian locomotor activity of Drosophila: they lengthen the period of circadian rhythms and increase arrhythmicity. Valproate exerts these effects in a weaker fashion than does lithium. We also tested the circadian alterations in multiple mutant lines of Drosophila bearing defects in the GSK-3 $\beta$ gene and other clock genes in response to lithium administration. We show that lithium partially rescues the shortening of circadian period when the GSK-3 $\beta$ gene is overexpressed only in specific circadian pacemaker neurons, thus implicating GSK-3 $\beta$ as a component in lithium's effect on the circadian oscillator. Moreover, lithium also lengthens the period in GSK-3 $\beta$ heterozygous mutants and doubletime long mutants. These results establish a basis for using Drosophila genetics to investigate more fully lithium and valproate action mechanisms.

Neuropsychopharmacology (2005) 30, 2216 -2224. doi:I 0. I 038/sj.npp. I 300764; published online I June 2005
\end{abstract}

Keywords: lithium; valproate; circadian rhythms; Drosophila; pharmacology; genetics

\section{INTRODUCTION}

Lithium and valproate are widely used medications in the treatment of bipolar disorder, a chronic and disabling illness for which no suitable animal models are available. The elucidation of action mechanisms of these drugs could lead to a better understanding of the etiopathogenesis of bipolar disorder. In addition, any improved insight could provide foundations for developing safer alternative therapeutic agents. Although research over several decades has identified many molecular targets of these drugs, these targets have not been studied in a behavioral paradigm using these drugs in a genetically tractable organism.

Many investigators have studied the effects of lithium (and, to a lesser extent, valproate) and there is a large body of literature reporting many acute, subacute, and chronic effects of lithium at the systems, cellular, and molecular levels (Manji et al, 1999b; Williams and Harwood, 2000;

\footnotetext{
*Correspondence: Dr ME Dokucu, Departments of Psychiatry and Anatomy and Neurobiology, Washington University School of Medicine, 660 S Euclid Ave, St Louis, MO 63110, USA, Tel: + I 314 747 4583, Fax: + I 314362 4247,

E-mail: dokucum@psychiatry.wustl.edu

Received 2 December 2004; revised 25 March 2005; accepted I April 2005

Online publication: 8 April 2005 at http://www.acnp.org/citations/ Npp040805040562/default.pdf
}

Shaldubina et al, 2001; Gurvich and Klein, 2002; Jope, 2003). The signaling molecules and pathways that have received more attention are phosphoinositide (PI)-protein kinase C (PKC) pathway, bcl-2/apoptosis machinery, and glycogen synthase kinase-3 $\beta$ (GSK-3 $\beta$ ). A recent report found that valproate also alters inositol metabolism by inhibiting myoinositol-1-phosphate synthase and causes inositol depletion by blocking de novo synthesis (Shaltiel et al, 2004). Manji and co-workers Chen et al, 1999b) have demonstrated in vivo that the apoptosis-inhibiting gene bcl-2 is induced both by lithium and valproate, assigning a potential neuroprotective role to these mood stabilizers. This group also reported that lithium treatment is associated with an increase in grey matter volume in patients (Moore et al, 2000). Importantly, valproate also inhibits GSK-3 $\beta$ (Chen et al, 1999a). As GSK-3 $\beta$ has proapoptotic effects, this is an intriguing connection of two signaling machineries.

Although GSK-3 $\beta$ inhibition by lithium was first reported in an early developmental context in Xenopus and Drosophila (Klein and Melton, 1996; Stambolic et al, 1996; Hedgepeth et al, 1997), the body of evidence is increasing that this may be valid also in the mammalian central nervous system (CNS) (Gould et al, 2004). It is significant that Drosophila shaggy mutants lacking GSK-3 $\beta$ activity display lengthening of the circadian period (Martinek et al, 2001). In humans, a single nucleotide polymorphism 
in the GSK-3 $\beta$ promoter region has been reported to correlate with the onset of bipolar disorder (Benedetti et al, 2004). Lithium signaling through GSK-3 $\beta$ has also been implicated in Alzheimer's disease pathology. Klein's group demonstrated that treatments with lithium or a small peptide that inhibits GSK-3 $\beta$ can block the production of $\mathrm{A} \beta$ peptides from amyloid precursor protein (Phiel et al, 2003).

Lithium treatment modifies circadian rhythms in humans and in most animals, primarily by lengthening the period of the cycle (Johnsson et al, 1980; Kripke and Wyborney, 1980; Welsh and Moore-Ede, 1990; Abe et al, 2000; Iwahana et al, 2004). Lithium directly inhibits GSK-3 $\beta$ activity in vitro (Ryves and Harwood, 2001) and in cell culture (Stambolic et al, 1996). But, the role of lithium's or valproate's inhibition of GSK-3 $\beta$ in circadian behavioral changes is unknown. Some authors have posited that the therapeutic action of lithium may be closely linked to its effect on circadian rhythms (Ikonomov and Manji, 1999; Manji and Lenox, 2000).

We aimed to extend investigations of mood-stabilizing drugs to a more genetically tractable organism and to apply them in a robust and phylogenetically conserved behavioral paradigm such as circadian behavior. Toward this aim, we studied the alterations in circadian behavior of wild-type and mutant Drosophila when they were chronically administered lithium and valproate.

Why use Drosophila in studying the action mechanisms of mood-stabilizing medications? The fly's small size and robustness, its reproduction cycle of 10 days, and 70 years of genetic research devoted to it make it an ideal genetic model organism. Although not nearly as complex in its behavioral repertoire as higher mammals, Drosophila is an excellent model organism that blends genetic accessibility and behavioral complexity. Drosophila has been successfully used in dissecting genetic components of behavioral paradigms including associative learning and memory (DeZazzo and Tully, 1995), substance abuse (Wolf, 1999; Wolf and Heberlein, 2003), circadian behavior (Wang and Sehgal, 2002), and recently sleep (Hendricks et al, 2001; Shaw et al, 2002). It is also very suitable for the study of gene interactions and epistasis. This has allowed researchers to use Drosophila in genetic screens to identify many additional components of signaling pathways such as the Ras pathway, which was first identified in mammalian model systems (Simon, 1994).

The level of detail achieved in defining the genetic components of the circadian machinery in Drosophila is unparalleled in any model system for any behavioral paradigm. This is at least partly due to its robustness. Circadian behavior is impaired in patients with psychiatric disorders, especially affective disorders (Klemfuss, 1992; Bunney and Bunney, 2000; Leibenluft and Frank, 2001). In addition, circadian behavior is very highly conserved across phylogeny. To this effect, a report has described a missense mutation in the human ortholog of the Drosophila period gene (hPer2) in familial advanced sleep phase syndrome patients (Toh et al, 2001). This finding attributes a human sleep behavior variant to a mutation in a clock gene (that was first identified in the fly) and suggests that at least one key behavioral gene has been conserved between insects and humans. Therefore, it is plausible that the signaling pathways targeted by mood-stabilizing agents are conserved by evolution.

Our results indicate that, consistent with the literature reporting lithium's effects on circadian behavior in mammals, lithium lengthens the period of circadian locomotor behavior in flies. Moreover, we observed that valproate shares this effect with lithium. We have tested GSK-3 $\beta$ (shaggy) mutants and also surveyed several other circadian clock mutants in our paradigm.

\section{MATERIALS AND METHODS}

\section{Fly Strains}

Flies were grown in nutritional media containing cornmeal, dextrose, agar, sodium potassium tartrate, calcium chloride, and the mold inhibitor Lexgard (Inolex, Philadelaphia, PA). The growth incubators were maintained at $25^{\circ} \mathrm{C}$ and at $60-$ $70 \%$ relative humidity. The flies were obtained either from Bloomington Drosophila Stock Center or were gifts from colleagues. We generated the pdfGAL4:UASsgg animals by crossing the pdfGAL4 carrying animals (Park et al, 2000) with UASsgg flies (obtained from Bloomington Stock Center) and choosing progeny that carried both constructs.

\section{Drug Administration and Lethality}

As lithium and valproate are both known teratogens, we chose to administer these drugs after the animals reached adulthood in order to avoid developmental abnormalities. Since circadian locomotor assays do not allow disturbance of the flies once the experiment is started, we administered lithium chloride (Sigma, St Louis) at $0.03-300 \mathrm{mM}$ concentrations chronically in the food. Food was not allowed to dry during preparation or afterwards. Likewise, we administered sodium valproate (Sigma, St Louis) at $0.1-25 \mathrm{mM}$ concentrations in fly food.

To determine the appropriate doses for behavioral analysis, we generated dose-lethality curves for lithium and valproate treatment. We mixed a $3-300 \mathrm{mM}$ range of lithium doses along with blue food coloring into the nutritional media. We placed 15-20 male and 15-20 female flies (age-matched) into each vial. Over the course of 8 days, we counted the number of dead flies daily. We also inspected the flies' abdomens for the presence of blue coloring to rule out death due to starvation. All flies had blue dye in their abdomens. We did not observe differences between male and female genders (data not shown). In addition, we compared the lethality caused by lithium chloride, lithium acetate, and lithium carbonate for the same doses and did not find any significant difference among these lithium salts (data shown for $\mathrm{LiCl}$ only). As seen in Figure 1a, the lethality of lithium up to $30 \mathrm{mM}$ in the food is quite low until the end of the experiment. Flies receiving lower concentrations of lithium continued to live for at least another 10 days (data not shown). As our behavioral assays last a maximum of 17 days, we did not conduct longer experiments for lethality. We conducted similar experiments testing the lethality of sodium valproate (Figure 1b). These experiments directed us to choose these doses for additional behavioral experiments: $10-30 \mathrm{mM}$ for lithium and $0.5 \mathrm{mM}$ for valproate. 

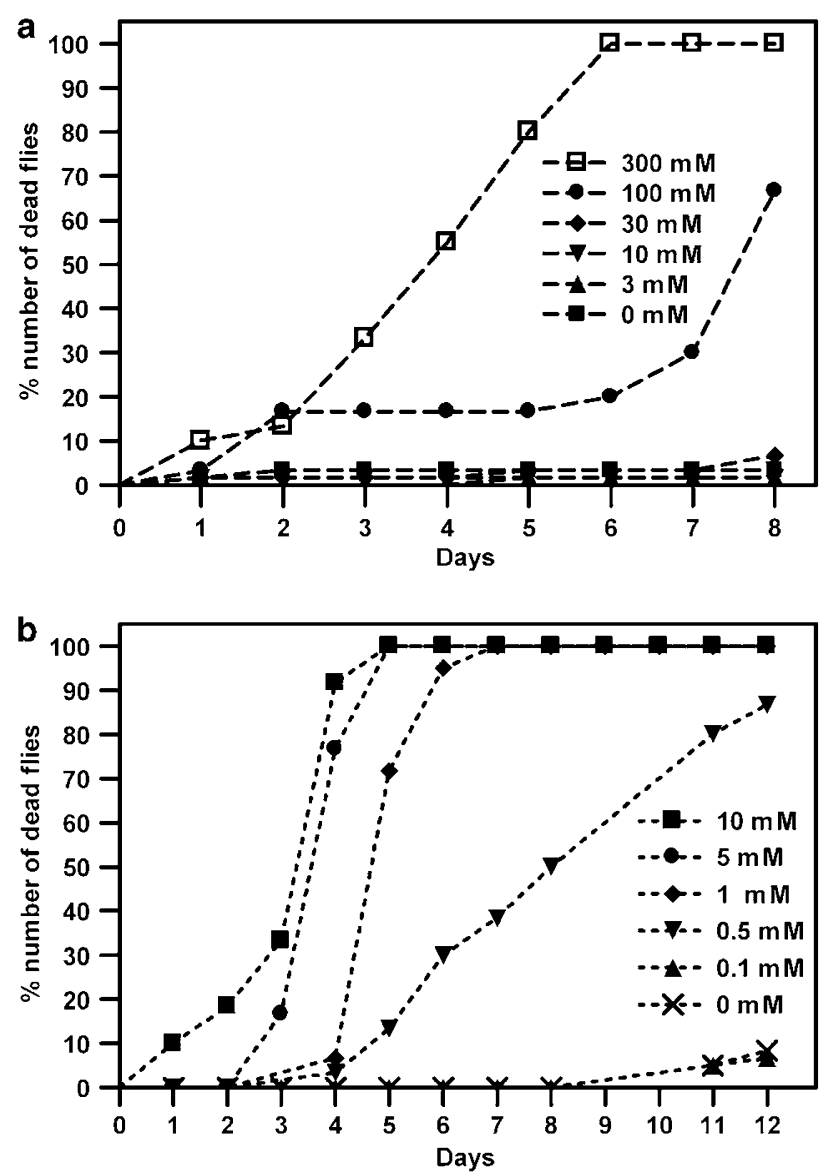

Figure I Lethality in populations of Drosophila due to administration of (a) lithium and (b) valproate at varying doses over time.

\section{Locomotor Behavior Assays}

Locomotor activity of flies was monitored as described by Taghert et al (2001). This was an automated assay. Briefly, flies were placed into $65 \mathrm{~mm}$ glass tubes midway through which a LED-emitted infrared beam vertically crosses the path of the fly's movement. Interruptions of this light beam were signaled to a personal computer (DAM software, Trikinetics Inc., Waltham, MA). An agar-sucrose-based food plug (with or without drug) was placed at one end of the glass tube. Once the animals were put into the Trikinetics monitors, they were not disturbed for the duration of the experiments. We typically monitored 1- to 3-day-old males at $25^{\circ} \mathrm{C}$ under $12 \mathrm{~h}$ light: $12 \mathrm{~h}$ dark conditions for 3-6 days and then released the animals into constant darkness (DD) for 9-14 days. The data were collected in half-hour bins and analyzed using the Brandeis Rhythm package (http://www.hawk.bcm.tcm.edu). We analyzed period lengths and rhythmicity of the animals with the $\chi^{2}$ periodogram analysis and maximum entropy spectral analysis (MESA). The rhythmicity of the animals was determined by the power criteria of the $\chi^{2}$ periodogram; we included only animals that were strongly rhythmic defined as having power $>10$ in our analyses. However, for determining arrhythmicity, we only counted animals that were strongly arrhythmic defined as nonsignificant $(p>0.01)$ by $\chi^{2}$ periodogram analysis. Statistical analyses were performed using Microsoft Excel subroutines and/or GraphPad Prism 4 software.

\section{Drug Level Assays}

To confirm and correlate observed behavioral alterations with drug dose, we measured lithium levels in whole flies using the ion-selective electrode method (Bertholf et al, 1988). Since the electrodes were calibrated to the electrolyte profile of human serum, we homogenized 10-20 flies into $200 \mu \mathrm{l}$ of fetal calf serum using a pestle following behavioral and uptake experiments. After centrifuging the solid matter down, we submitted the supernatants to Washington University Medical Center Clinical Laboratories for testing. We included standard solution samples as an internal quality control measure.

\section{RESULTS}

Previous work had shown that lithium could be administered through fly food reliably and without lethality (Williamson, 1982; Xia et al, 1997). Since these experiments were performed for short durations and since our circadian locomotor activity assays involve weeks, we tested the feasibility of longer duration of drug administration in our paradigm. We report here that lithium and valproate can be reliably given to Drosophila and that they lengthen the circadian period of locomotor activity in a Canton S laboratory strain (wild type) flies. A preliminary aim was the preparatory studies of choosing the lithium salt optimal for survival and defining dosages of administration of lithium to flies. The lithium levels measured in the fly leading to behavioral change are also within the 'physiological' range as reported in human and rodent literature. Lithium increased the number of arrhythmic flies in a dosedependent manner, most likely by decreasing the amplitude of activity levels. In addition, we have identified changes in the circadian behavior of two GSK-3 $\beta$ mutant strains: pdfGAL4:UASsgg flies (overexpressing GSK-3 $\beta$ ) and sgg/ FM6 (heterozygous null mutant). We also observed circadian alterations by lithium administration in the behavior of doubletime [L] (the long-period allele) homozygous circadian mutants.

\section{Lithium Uptake Over Time}

Based on the lethality experiments, we were confident that flies were taking up lithium. Next, we asked whether this could be detected using standard clinical laboratory assays in order to correlate drug levels in the body with concentration in food or to correlate with behavior. We placed flies in vials including food with a range of lithium concentrations. We collected flies every 3 days and froze them. At the end of 15 days, we measured lithium levels. Figure 2 shows the uptake of lithium over 2 weeks. This experiment shows that the lithium levels in whole flies track the lithium concentration in the fly food. A two-way ANOVA test showed that there is a strong correlation of lithium levels with the concentration in food $(p<0.0001)$ and a weak correlation with duration $(p<0.02)$. The lethality observed in this experiment is consistent with that observed in others (ie Figure 1a). We calculated a range of 


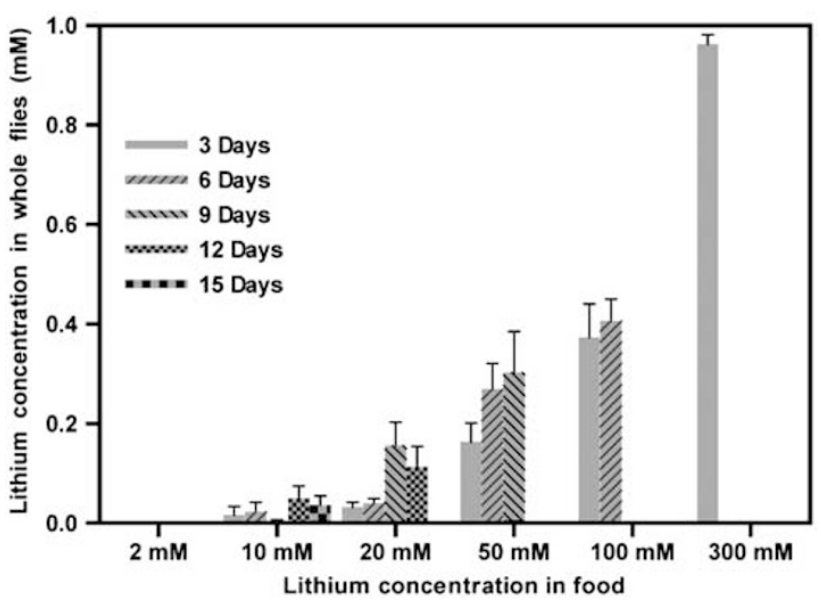

Figure 2 Lithium uptake by Drosophila as a function of lithium concentration in fly food. Lithium levels on $y$-axis reflect the readings for I0 flies homogenized as described in Materials and Methods. The data points missing are due to undetectable levels of lithium (for $2 \mathrm{mM}$ ) or no flies remaining alive at that date $(6,9,12$, and 15 days for $300 \mathrm{mM} ; 9,12$, and 15 days for $100 \mathrm{mM}$; 12 and 15 days for $50 \mathrm{mM}$; and 15 days for $20 \mathrm{mM}$ ) (error bars indicate SEM).

concentration from 0.2 to $14 \mathrm{mM}$ per fly based on the average volume of the animals and number used in the assays. We observed behavioral effects at the lower end of this range (which is comparable to 'therapeutic' levels recorded in mammalian studies) and lethality at the higher end.

\section{Lithium Levels after Behavioral Experiment}

We asked whether flies uptake lithium similarly at the end of the locomotor behavioral assay. Following a 15-day experiment that monitored circadian locomotor output, we collected 20 live flies per dosage in duplicate and measured lithium levels (using the ion-selective electrode method). Figure 3 reveals that the fly lithium concentration follows food lithium concentration (for duplicate samples) in this experiment, as observed in the uptake experiment described previously. We also tested the hypothesis that lithium levels in the fly correlate with the increase in the period. After the behavioral experiment, we grouped flies displaying larger increase in circadian period and flies showing small or no increase in circadian period. We assayed these two groups of flies and did not detect a significant difference in lithium levels (data not shown) between groups.

\section{Lithium and Valproate's Effects on the Circadian Locomotor Behavior of Adult Wild-Type Drosophila}

We conducted several experiments analyzing circadian locomotor behavior parameters in adult wild-type (Canton S) Drosophila that received a wide dose range (0.03$300 \mathrm{mM}$ ) of lithium in the nutritional media. We used 40-250 adult flies (typically 1- to 3-day-old males) per experimental condition and obtained similar results across experimental replicates (aggregate results are reported). Although there is some evidence that lithium may regulate light sensitivity (Seggie et al, 1989; Duncan et al, 1998), our initial focus was the analysis of its effect on the free-running

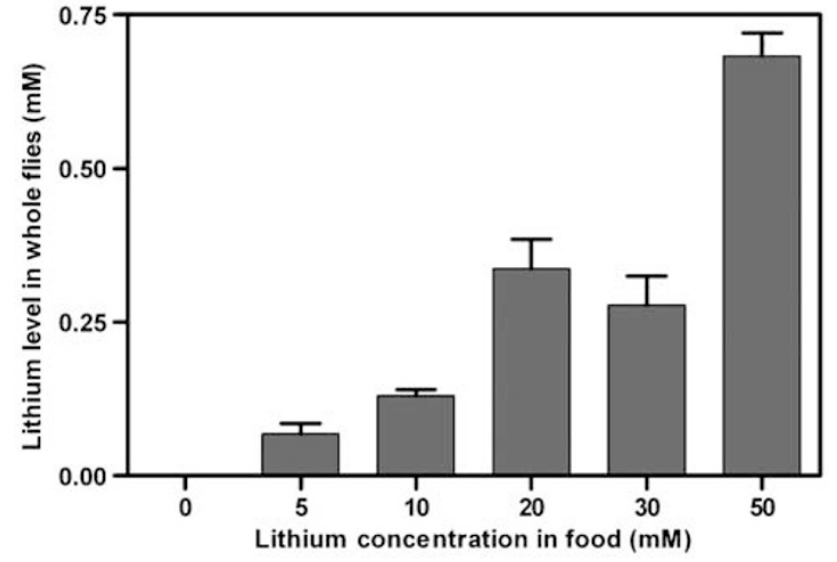

Figure 3 Lithium uptake by Drosophila following a 15-day behavioral experiment. Lithium levels on the $y$-axis reflect the readings for 20 flies per value (error bars indicate SEM).
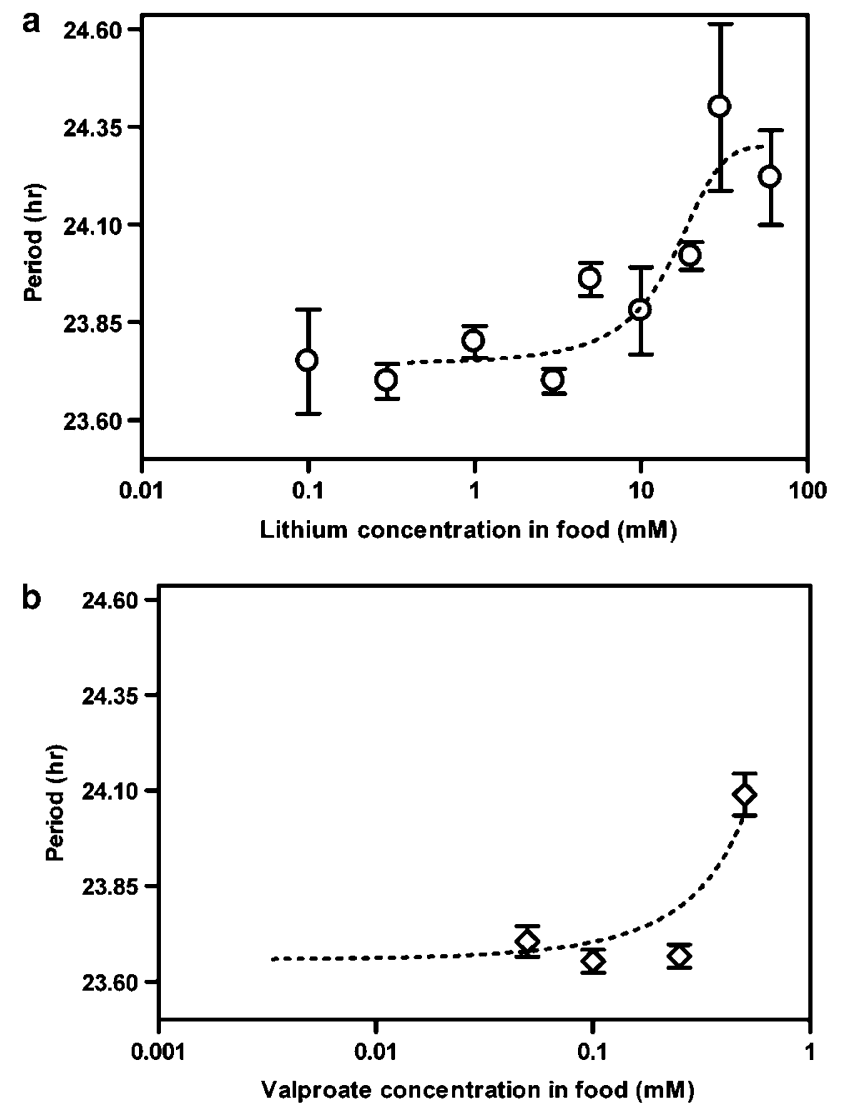

Figure 4 Increase in the period of circadian locomotor activity with the administration of (a) lithium and (b) valproate to wild-type Drosophila. $x$ axis is logarithmic (error bars indicate SEM) For statistical summaries, see Table I.

clock (ie DD conditions). We observed that lithium induced a significant increase in the period of the free-running locomotor rhythms of the fly. This trend displayed a narrow 'therapeutic' window such that, with higher than $30 \mathrm{mM}$ concentrations of lithium in the food, the number of flies that died before the experiment ended increased dramatically. Figure $4 \mathrm{a}$ displays the dose-response curve for 
Table I Increase in the Period of Circadian Locomotor Activity in dbt[L] Flies after Lithium Administration (Bottom Two Rows), Significant as Indicated by Student's Two-Tailed t-Test

Test and significance

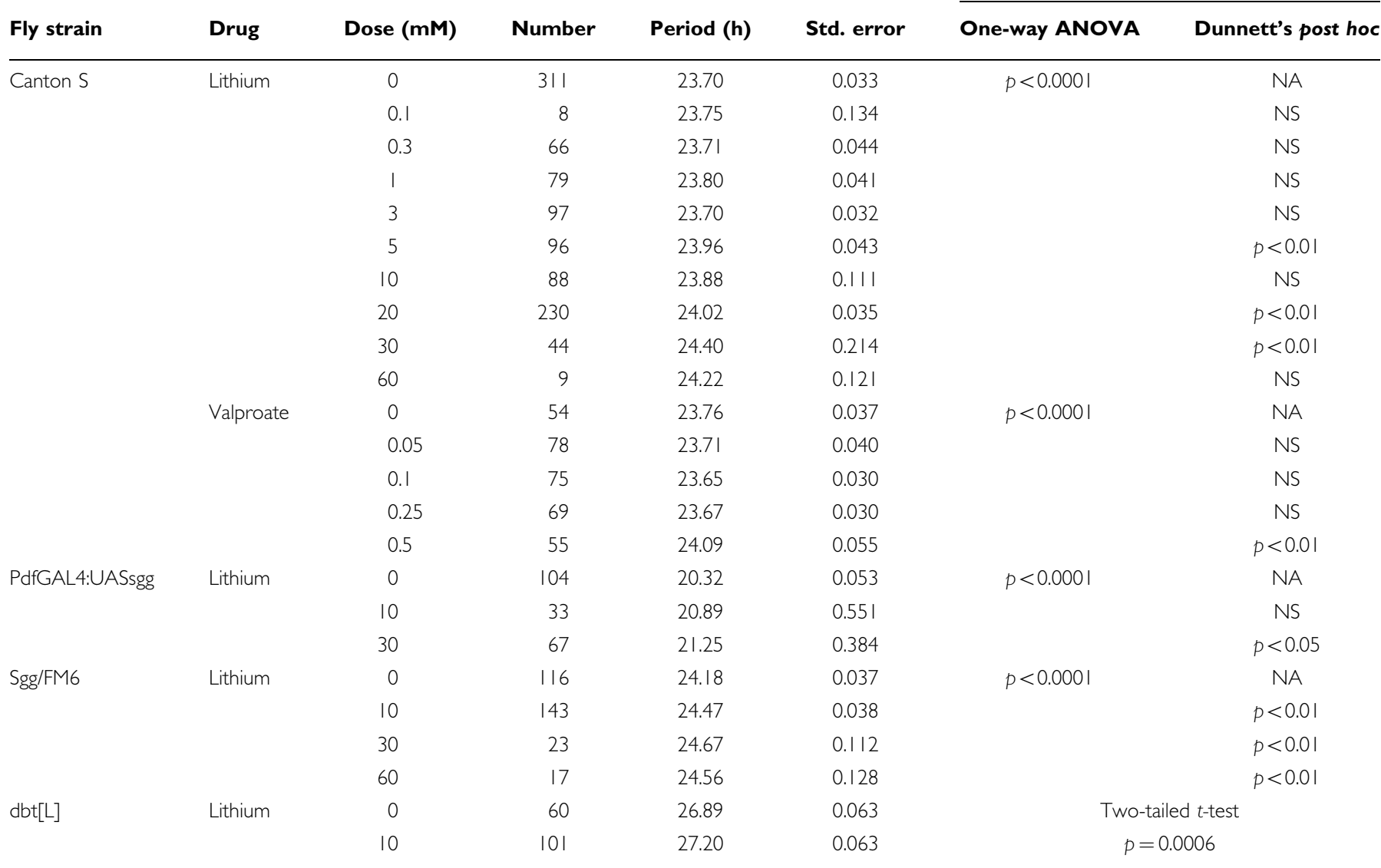

In addition, statistical summary (ie of experiments in Figures 4 and 5) is given. For these experiments, the one-way ANOVAs were followed by Dunnett's post hoc test between controls and various doses of drugs. (For drug doses for which multiple experiments were performed, we report combined results.) NA, not applicable; NS, not significant.

circadian locomotor activity period. Table 1 shows the statistical profile of the data that generated the graph in Figure 4a. Figure 7 shows double-plotted actograms from individual wild-type flies that reflect the mean behavior observed by lithium administration. We also observed that, similar to the results with lithium administration, valproate caused a more modest but significant lengthening of the circadian period after administering it to adult wild-type Drosophila at $0.5 \mathrm{mM}$ in the food. Figure $4 \mathrm{~b}$ displays the dose-response curve for this effect. It is cutoff abruptly due to higher toxicity of valproate for Drosophila. Thus, we show for the first time a shared effect of lithium and valproate on the circadian behavior of an animal.

\section{Lithium's Effects on the Circadian Behavior of Adult Mutant Drosophila}

GSK-3 $\beta$ (shaggy) mutants were isolated through an EPelement insertion screen using timeless-GAL4 as a driver, which misexpressed many genes in the neurons that expressed the timeless clock gene (Martinek et al, 2001). We tested flies heterozygous for a null GSK-3 $\beta$ mutation, flies that expressed GSK- $3 \beta$ ubiquitously when given heat treatments, and flies that expressed GSK-3 $\beta$ under $p d f$ promoter control. Since GSK-3 $\beta$ null mutations produce lethality in flies, it is not possible to test the homozygous loss-of-function phenotype of this mutation directly. Young and co-workers (Martinek et al, 2001) devised a recovery design with which they rescued GSK-3 $\beta$ expression during metamorphosis. These flies subsequently had very little GSK-3 $\beta$ function left and they displayed very long periods. In our lithium administration paradigm, the yield of rescued loss-of-function animals (hypomorphs) was too low and we did not see any change in response to lithium treatment (data not shown). Therefore, we chose to analyze the GSK-3 $\beta$ heterozygote mutants. Without lithium treatment, heterozygote mutants display a period of $24.2 \mathrm{~h}$ (wild type is approximately $23.7 \mathrm{~h}$ ) (Table 1 ). When given lithium (10-60 mM), these flies displayed lengthening of their period (Figure 5b). We also generated mutants that overexpress GSK-3 $\beta$ (ie pdfGAL4:UASsgg mutants; see the Fly Strains section). These mutants express GSK-3 $\beta$ specifically in the clock cells of the Drosophila brain (in addition to the natively expressed protein). In testing these animals, we found a significant and largest increase in period where lithium partially rescues the short-period 

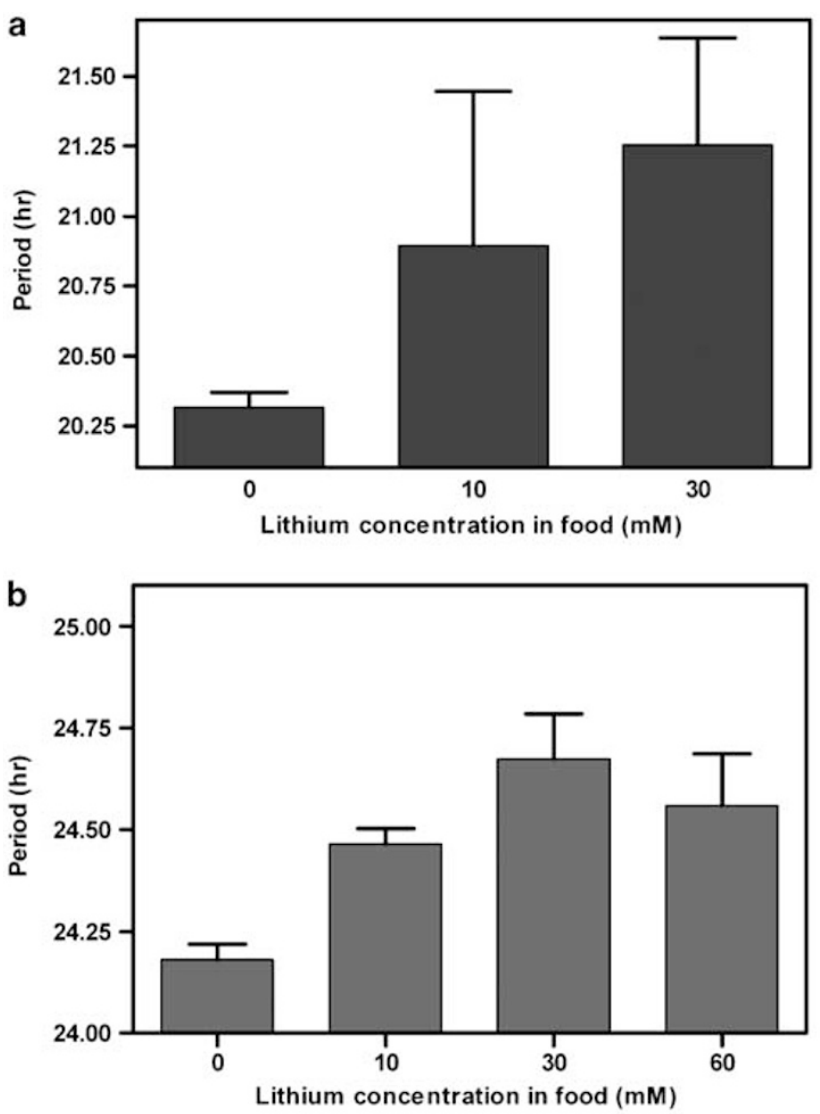

Figure 5 Increase in the period of circadian locomotor activity after administration of lithium to mutant Drosophila (a) when expressing excess GSK-3 $\beta$ in clock neurons (pdfGAL4:UASsgg), and (b) carrying a heterozygous null mutation of GSK-3 $\beta$ (sgg/FM6). For statistical summaries, see Table I.

mutant phenotype (ie increases the shortened period bringing it closer to wild-type period) (Figure 5a). We have also tested flies bearing various mutations in the genes coding for the critical proteins of the core molecular clock in the fly: period (short- and long-period alleles), and timeless and doubletime (short- and long-period alleles) genes. These mutants are described by Williams and Sehgal (2001). The doubletime [L] homozygous mutants responded to lithium by showing a statistically significant lengthening of their period ( $t$-test with Welch correction, two-tailed $p=0.0006$ ) (Table 1). Figure 7 shows sample double-plotted actograms from individual mutant flies that reflect the mean behavior observed in controls and by lithium administration.

\section{Lithium and Valproate Increase Arrhythmicity in Flies}

We also analyzed the effect of lithium and valproate on the behavioral rhythmicity of the animal. We observed that both lithium and valproate treatment increase the number of arrhythmic animals in prolonged DD as determined by lack of significant rhythmic locomotor activity ( $\chi^{2}$ periodogram test, $p<0.01$ ) (Figure 6). Similar to the effect on circadian period, the effect of lithium on increasing the number of arrhythmic flies was more pronounced than that of valproate.
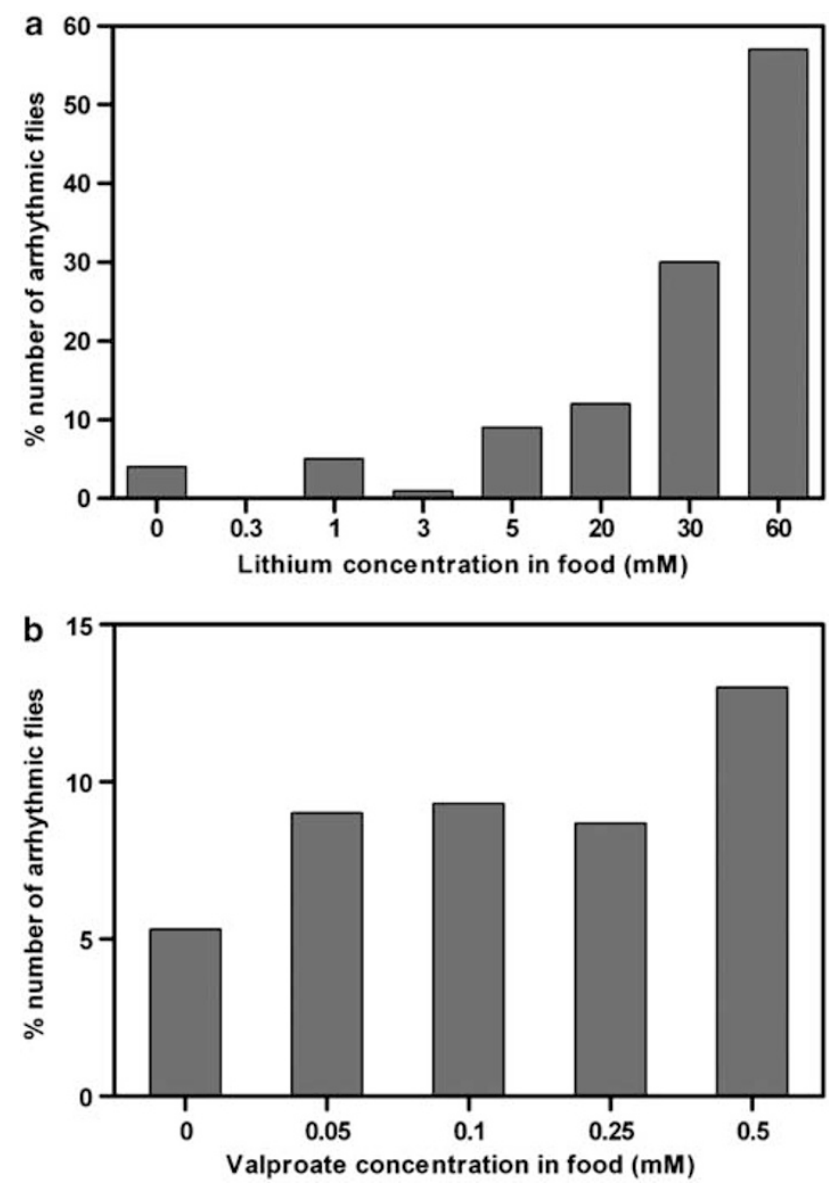

Figure 6 Increase in the percentage of the number of flies that display locomotor arrhythmia after administration of (a) lithium and (b) valproate. See Materials and Methods for criteria of rhythmic behavior.

\section{DISCUSSION}

We have demonstrated shared effects on circadian behavior of two mood stabilizers, lithium and valproate, using the model organism Drosophila as a prelude to developing it as a system for identifying novel molecular targets for the mood stabilizers. We also implicate GSK-3 $\beta$ as one of the key components in lithium's effect on circadian behavior.

Our data indicated that lithium lengthens free-running circadian period in wild-type flies. While this manuscript was being prepared, this same finding was reported by others (Padiath et al, 2004). Our data indicate that overexpression of GSK-3 $\beta$ in clock cells of the brain increases lithium's effect on the circadian period by approximately two-fold. This is consistent with (but not conclusive for) lithium exerting this type of effect by signaling mainly through GSK-3 $\beta$ enzyme. It is currently unknown whether the behavioral phenotype observed is a result of direct inhibition by lithium, as reported in vitro or in cell culture. However, without data obtained from null mutants of GSK-3 $\beta$, we cannot rule out other signaling pathways. It is also of interest that lithium can increase period in doubletime long mutants that already have a very long period, suggesting that if there is a ceiling effect, it is not dependent on the length of the period but may involve dose limitations or effect size due to toxicity. Lithium's lack 

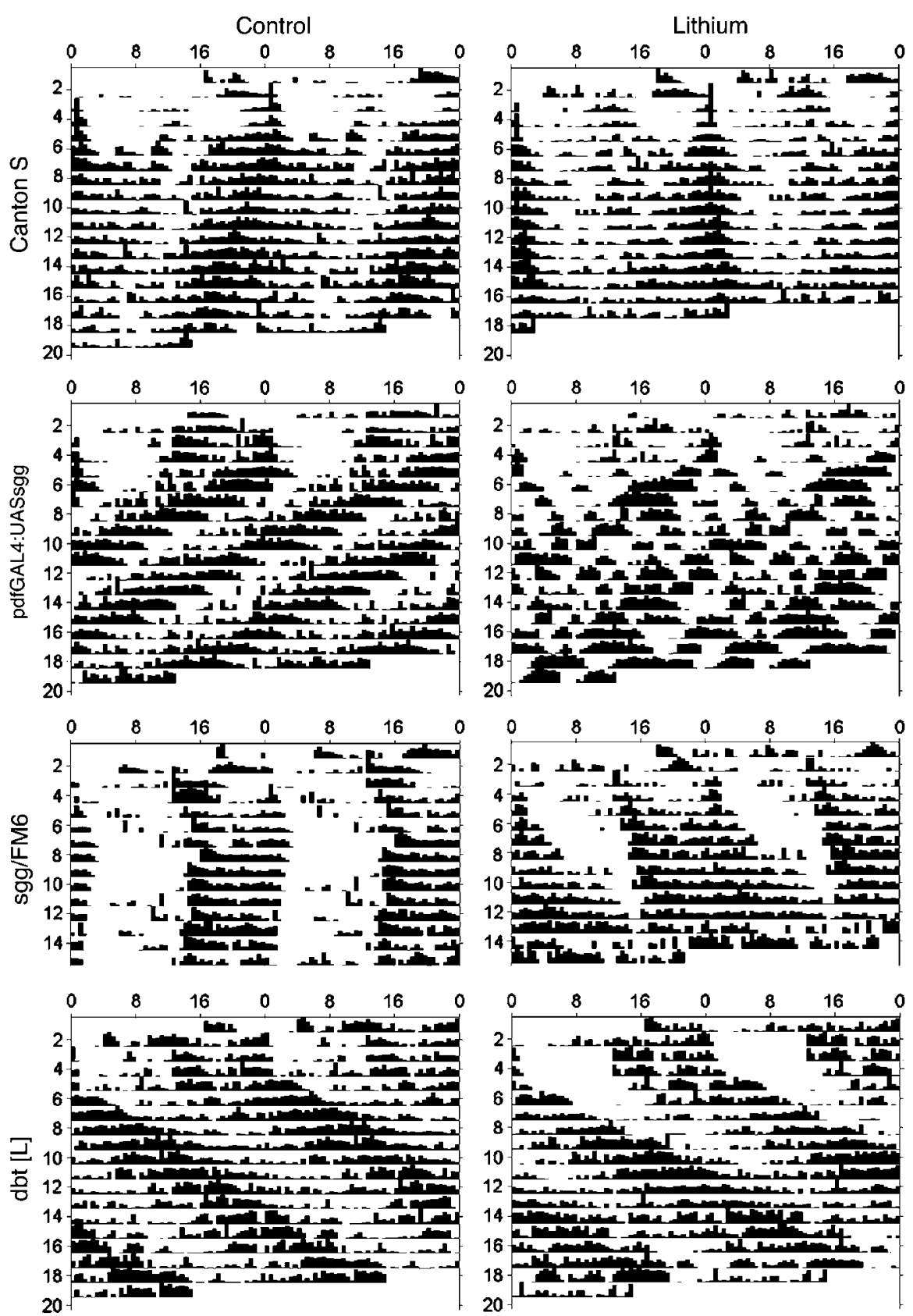

Figure 7 Double-plotted locomotor activity graphs of sample individual flies. For each graph, the $x$-axis denotes $48 \mathrm{~h}$ of time and $y$-axis denotes level of activity on each day of the experiment in a raster style (top to bottom, left to right). The second $24 \mathrm{~h}$ of data on the previous day of activity are replotted in the beginning of the next day's line to facilitate the perception of the activity patterns by the viewer. The alignment of the activity peaks correlates with the circadian period (peaks vertically aligned $=24 \mathrm{~h}$ ). The left-hand panels are wild-type (Canton S) and mutant flies without lithium administration. The righthand panels are wild-type and mutant flies that received lithium at $30 \mathrm{mM}$ dose with the exception of the bottom two panels where the flies received $10 \mathrm{mM}$ lithium.

of effect on the circadian behavior of other clock mutants that we tested (especially in short-period allele of doubletime) is difficult to interpret, as we have not performed experiments with wider dose ranges with these mutants yet. By studying double mutants of doubletime and shaggy, it could be possible to define the epistatic relationships of these two genes with relationship to circadian period changes induced by lithium. Based on the circadian phenotype of Drosophila mutants lacking GSK-3 $\beta$ activity (Martinek et al, 2001), and based on our data and that of others (Padiath et al, 2004), we propose that lithium increases the circadian period by inhibiting GSK-3 $\beta$. These experiments strongly suggest that the fly's response to lithium is similar to that of rodents as reported before (Abe et al, 2000; Iwahana et al, 2004).

It is currently unclear how serum lithium concentrations in humans translate to the brain lithium levels (Kato et al, 1992; Gonzalez et al, 1993; Plenge et al, 1994). Although it is technically possible to measure lithium 
levels in the heads of the flies, for economic reasons, we chose to measure levels in whole flies. Our calculations of lithium levels in individual flies were consistent with levels in humans and rodents. It is also important to note that we observed statistically significant circadian behavioral effects at the $5 \mathrm{mM}$ dose in food, although lithium concentration at $10 \mathrm{mM}$ dose was barely detectable by ion-selective electrodes in the uptake experiment. This could be due to the low sensitivity of the instrument or higher levels of lithium in the CNS of the fly. In addition, the lethality observed in the uptake experiments (Figure 2) was slightly higher for similar doses in the lethality experiment (Figure 1a). To address this, we also measured the lithium levels following a 2-week behavioral experiment and observed that, within experiments, food concentration of lithium correlates well with the lithium levels in whole flies.

Although valproate use in bipolar disorder is as common as lithium use, much less is known of valproate's mechanism of action either as an anticonvulsant or as a mood stabilizer. The neurotransmitter that is most likely involved in valproate's antiseizure action is GABA: valproate increases GABA in synapses and inhibits its catabolism. More intriguingly, valproate alters PI signaling and GSK-3 $\beta$ activity in a similar way but likely via action on different molecules (Chen et al, 1999a). Other action modalities valproate appears to share with lithium are induction of GluR-1, ERK signaling pathway, bcl-2, AP-1 transcription factor complex, and protein kinase C (Chen et al, 1999b; Manji et al, 1999a; Manji and Chen, 2002; Du et al, 2003; Einat et al, 2003). The overlapping action modalities at the signaling pathways and molecules make it likely that valproate may have similar effects on circadian physiology to those exerted by lithium. Manji et al (2001) and Harwood (2003) have posited that valproate and lithium studied together may lead to fewer false-positive leads, as lithium is a considerably 'dirtier' drug than valproate. At our current level of understanding, this is a rational reduction strategy. Harwood and co-workers have extended this strategy to include carbamazepine and have shown in cell cultures that all three mood stabilizers inhibit the collapse of growth cones and increase growth cone area. This likely happens independently of GSK-3 $\beta$ or histone deacylase inhibition but is possibly dependent on inositol depletion (Williams et al, 2002). We have extended the above cellular and molecular studies by testing valproate effects on circadian rhythms and shown that, similar to lithium, valproate lengthens the circadian period and the rate of arrhythmicity albeit with smaller effect size. This may reflect overlapping but not identical action pathways for these structurally very dissimilar drugs. Lithium may be affecting additional targets that influence circadian behavior and therefore contribute to a larger effect size. Other possibilities include a ceiling effect for valproate due to higher toxicity, that is, low valproate levels in the brain secondary to peripheral toxicity leading to early death that is different from what is clinically observed in humans. We are pursuing uptake studies with valproate administration.

Future experiments will include utilizing Drosophila via genetic screens to identify other molecular targets for mood-stabilizing drugs in a behavioral framework.

\section{ACKNOWLEDGEMENTS}

MED received a Young Investigator Award from NARSAD and an NRSA from NIMH. PHT received funding from NIMH (RO1MH067122). We are indebted to Mitch Scott for his help in clinical assays. Michael Young kindly shared Drosophila strains generated in his laboratory. We thank Amita Sehgal, Taghert laboratory members, and 'Clocks Club' participants for valuable discussions. We appreciated the technical help of Weihua Li and Molly Peck.

\section{REFERENCES}

Abe M, Herzog ED, Block GD (2000). Lithium lengthens the circadian period of individual suprachiasmatic nucleus neurons. Neuroreport 11: 3261-3264.

Benedetti F, Bernasconi A, Lorenzi C, Pontiggia A, Serretti A, Colombo C et al (2004). A single nucleotide polymorphism in glycogen synthase kinase 3-beta promoter gene influences onset of illness in patients affected by bipolar disorder. Neurosci Lett 355: $37-40$.

Bertholf RL, Savory MG, Winborne KH, Hundley JC, Plummer GM, Savory J (1988). Lithium determined in serum with an ionselective electrode. Clin Chem 34: 1500-1502.

Bunney WE, Bunney BG (2000). Molecular clock genes in man and lower animals: possible implications for circadian abnormalities in depression. Neuropsychopharmacology 22: 335-345.

Chen G, Huang LD, Jiang YM, Manji HK (1999a). The moodstabilizing agent valproate inhibits the activity of glycogen synthase kinase-3. J Neurochem 72: 1327-1330.

Chen G, Zeng WZ, Yuan PX, Huang LD, Jiang YM, Zhao ZH et al (1999b). The mood-stabilizing agents lithium and valproate robustly increase the levels of the neuroprotective protein bcl-2 in the CNS. $J$ Neurochem 72: 879-882.

DeZazzo J, Tully T (1995). Dissection of memory formation: from behavioral pharmacology to molecular genetics. Trends Neurosci 18: $212-218$.

Du J, Gray NA, Falke C, Yuan P, Szabo S, Manji HK (2003). Structurally dissimilar antimanic agents modulate synaptic plasticity by regulating AMPA glutamate receptor subunit GluR1 synaptic expression. Ann NY Acad Sci 1003: 378-380.

Duncan WC, Johnson Jr KA, Wehr TA (1998). Decreased sensitivity to light of the photic entrainment pathway during chronic clorgyline and lithium treatments. J Biol Rhythms 13: 330-346.

Einat H, Manji HK, Gould TD, Du J, Chen G (2003). Possible involvement of the ERK signaling cascade in bipolar disorder: behavioral leads from the study of mutant mice. Drug News Perspect 16: 453-463.

Gonzalez RG, Guimaraes AR, Sachs GS, Rosenbaum JF, Garwood M, Renshaw PF (1993). Measurement of human brain lithium in vivo by $\mathrm{MR}$ spectroscopy. AJNR Am J Neuroradiol 14: 1027-1037.

Gould TD, Chen G, Manji HK (2004). In vivo evidence in the brain for lithium inhibition of glycogen synthase kinase-3. Neuropsychopharmacology 29: 32-38.

Gurvich N, Klein PS (2002). Lithium and valproic acid: parallels and contrasts in diverse signaling contexts. Pharmacol Ther 96: $45-66$.

Harwood AJ. (2003). Neurodevelopment and mood stabilizers. Curr Mol Med 3: 472-482.

Hedgepeth CM, Conrad LJ, Zhang J, Huang HC, Lee VM, Klein PS (1997). Activation of the Wnt signaling pathway: a molecular mechanism for lithium action. Dev Biol 185: 82-91.

Hendricks JC, Williams JA, Panckeri K, Kirk D, Tello M, Yin JC et al (2001). A non-circadian role for cAMP signaling and CREB 
activity in Drosophila rest homeostasis. Nat Neurosci 4: $1108-1115$

Ikonomov OC, Manji HK (1999). Molecular mechanisms underlying mood stabilization in manic-depressive illness: the phenotype challenge. Am J Psychiatry 156: 1506-1514.

Iwahana E, kiyama MA, Miyakawa K, Uchida A, Kasahara J, Fukunaga $\mathrm{K}$ et al (2004). Effect of lithium on the circadian rhythms of locomotor activity and glycogen synthase kinase-3 protein expression in the mouse suprachiasmatic nuclei. Eur J Neurosci 19: 2281-2287.

Johnsson A, Engelmann W, Pflug B, Klemke W (1980). Influence of lithium ions on human circadian rhythms. Z Naturforsch [C] 35: 503-507.

Jope RS (2003). Lithium and GSK-3: one inhibitor, two inhibitory actions, multiple outcomes. Trends Pharmacol Sci 24: 441-443.

Kato T, Takahashi S, Inubushi T (1992). Brain lithium concentration by $7 \mathrm{Li}$ - and $1 \mathrm{H}$-magnetic resonance spectroscopy in bipolar disorder. Psychiatry Res 45: 53-63.

Klein PS, Melton DA (1996). A molecular mechanism for the effect of lithium on development. Proc Natl Acad Sci USA 93: 8455-8459.

Klemfuss H. (1992). Rhythms and the pharmacology of lithium. Pharmacol Ther 56: 53-78.

Kripke DF, Wyborney VG (1980). Lithium slows rat circadian activity rhythms. Life Sci 26: 1319-1321.

Leibenluft E, Frank E (2001). Circadian rhythms in affective disorders. In: Takahashi JS, Turek FW, Moore RY (eds). Circadian Clocks. Kluwer Academic/Plenum Publishers: New York. Vol 12, pp 625-644.

Manji HK, Bebchuk JM, Moore GJ, Glitz D, Hasanat KA, Chen G (1999a). Modulation of CNS signal transduction pathways and gene expression by mood-stabilizing agents: therapeutic implications. J Clin Psychiatry 60(Suppl 2): 27-39 discussion 40-41, 113-116.

Manji HK, Chen G (2002). PKC, MAP kinases and the bcl-2 family of proteins as long-term targets for mood stabilizers. $\mathrm{Mol}$ Psychiatry 7(Suppl 1): S46-S56.

Manji HK, Lenox RH (2000). The nature of bipolar disorder. J Clin Psychiatry 61(Suppl 13): 42-57.

Manji HK, Moore GJ, Chen G (1999b). Lithium at 50: have the neuroprotective effects of this unique cation been overlooked? Biol Psychiatry 46: 929-940.

Manji HK, Moore GJ, Chen G (2001). Bipolar disorder: leads from the molecular and cellular mechanisms of action of mood stabilizers. Br J Psychiatry Suppl 41: s107-s119.

Martinek S, Inonog S, Manoukian AS, Young MW (2001). A role for the segment polarity gene shaggy/GSK-3 in the Drosophila circadian clock. Cell 105: 769-779.

Moore GJ, Bebchuk JM, Wilds IB, Chen G, Manji HK, Menji HK (2000). Lithium-induced increase in human brain grey matter. Lancet 356: 1241-1242.

Padiath QS, Paranjpe D, Jain S, Sharma VK (2004). Glycogen synthase kinase 3 beta as a likely target for the action of lithium on circadian clocks. Chronobiol Int 21: 43-55.

Park JH, Helfrich-Forster C, Lee G, Liu L, Rosbash M, Hall JC (2000). Differential regulation of circadian pacemaker output by separate clock genes in Drosophila. Proc Natl Acad Sci USA 97: 3608-3613.
Phiel CJ, Wilson CA, Lee VM, Klein PS (2003). GSK-3alpha regulates production of Alzheimer's disease amyloid-beta peptides. Nature 423: 435-439.

Plenge P, Stensgaard A, Jensen HV, Thomsen C, Mellerup ET, Henriksen O (1994). 24-hour lithium concentration in human brain studied by Li-7 magnetic resonance spectroscopy. Biol Psychiatry 36: 511-516.

Ryves WJ, Harwood AJ (2001). Lithium inhibits glycogen synthase kinase- 3 by competition for magnesium. Biochem Biophys Res Commun 280: 720-725.

Seggie J, Carney PA, Parker J, Grof E, Grof P (1989). Effect of chronic lithium on sensitivity to light in male and female bipolar patients. Prog Neuropsychopharmacol Biol Psychiatry 13: $543-549$.

Shaldubina A, Agam G, Belmaker RH (2001). The mechanism of lithium action: state of the art, ten years later. Prog Neuropsychopharmacol Biol Psychiatry 25: 855-866.

Shaltiel G, Shamir A, Shapiro J, Ding D, Dalton E, Bialer M et al (2004). Valproate decreases inositol biosynthesis. Biol Psychiatry 56: $868-874$.

Shaw PJ, Tononi G, Greenspan RJ, Robinson DF (2002). Stress response genes protect against lethal effects of sleep deprivation in Drosophila. Nature 417: 287-291.

Simon MA. (1994). Signal transduction during the development of the Drosophila R7 photoreceptor. Dev Biol 166: 431-442.

Stambolic V, Ruel L, Woodgett JR (1996). Lithium inhibits glycogen synthase kinase- 3 activity and mimics wingless signalling in intact cells. Curr Biol 6: 1664-1668.

Taghert PH, Hewes RS, Park JH, O'Brien MA, Han M, Peck ME (2001). Multiple amidated neuropeptides are required for normal circadian locomotor rhythms in Drosophila. J Neurosci 21: 6673-6686.

Toh KL, Jones CR, He Y, Eide EJ, Hinz WA, Virshup DM et al (2001). An hPer2 phosphorylation site mutation in familial advanced sleep phase syndrome. Science 291: 1040-1043.

Wang GK, Sehgal A (2002). Signaling components that drive circadian rhythms. Curr Opin Neurobiol 12: 331-338.

Welsh DK, Moore-Ede MC (1990). Lithium lengthens circadian period in a diurnal primate, Saimiri sciureus. Biol Psychiatry 28: 117-126.

Williams JA, Sehgal A (2001). Molecular components of the circadian system in Drosophila. Annu Rev Physiol 63: 729-755.

Williams RS, Cheng L, Mudge AW, Harwood AJ (2002). A common mechanism of action for three mood-stabilizing drugs. Nature 417: 292-295.

Williams RS, Harwood AJ (2000). Lithium therapy and signal transduction. Trends Pharmacol Sci 21: 61-64.

Williamson RL. (1982). Lithium stops hereditary shuddering in Drosophila melanogaster. Psychopharmacology (Berl) 76: 265268.

Wolf FW, Heberlein U (2003). Invertebrate models of drug abuse. J Neurobiol 54: 161-178.

Wolf ME (1999). Cocaine addiction: clues from Drosophila on drugs. Curr Biol 9: R770-R772.

Xia S, Liu L, Feng C, Guo A (1997). Drug disruption of short-term memory in Drosophila melanogaster. Pharmacol Biochem Behav 58: $727-735$. 